\title{
Pardee wave in an infant: what is the etiology?
}

\author{
S. tilouche ${ }^{1}, \underline{\text { S. ghorbel }}^{1}$, M. jedidi ${ }^{2}$, A. tej ${ }^{1}$, R. kebaili ${ }^{1}$, J. bouguila ${ }^{1}$, N. kahloull ${ }^{1}$, L. \\ boughamoura ${ }^{1}$. \\ ${ }^{1}$ Farhat Hached hospital, pediatric, sousse, Tunisia. \\ ${ }^{2}$ Farhat Hached hospital, legal departement, sousse, Tunisia.
}

\section{Introduction:}

Usually, in adults care, ST segment abnormality and accurate biomarkers of cardiac injury specially troponin, confirm myocardial infraction and allow interventional catheterization . In children, the most common cause is myocarditis or cardiomyopathy, whereas coronary-related ischemia is rare

Through the observation of an infant and through a review of literature, we recall the clinical and evolutionary features of myocarditis.
Troponin was at 0.81 [normal value $<0,25 \mathrm{ng} / \mathrm{mL}$ ]. on admission and then at $1.92 \mathrm{ng} / \mathrm{ml}$ at the $2 \mathrm{nd}$ hour. CPK and LDH were high. The infant required mechanical ventilation, he had received milrinone and veinoglobulins. The infant died in the third day after admission due to cardiogenic shock and a forensic autopsy was performed because of unexplained cause of death. The autopsy had concluded to myocarditis.

\section{Observation:}

Infant 9 months old, was admitted for dyspnea and cough. On examination, she had a pulse of $180 / \mathrm{min}$, a blood pressure of $70 / 40 \mathrm{mmHg}$ and a respiratory rate of $48 / \mathrm{min}$ with chest indrawing. The temperature was $38.9^{\circ} \mathrm{C}$. At the 20th minute of hospitalization, the infant presented with ventricular tachycardia and cardiorespiratory arrest. The cardiac arrest was recovered after 5 minutes of resuscitation, but the child subsequently presented an ST overcompensation and then a Pardee wave at the ECG.

Ultrasound showed a dilated left ventricle with impaired global systolic function with a $22 \%$ ejection fraction.

\section{Discussion}

Acute myocarditis in children is a rare condition with variable clinical expression. The mode of revelation is dominated by respiratory and / or digestive symptoms occurring during or after a flu-like episode in a child with no history of cardiology.

The fulminant forms are exceptional. Their diagnosis, unlike acute myocarditis, is essentially clinical. Non-specific electrocardiographic abnormalities such as ST under-shift, T-wave inversion and QRS microvoltage are common: $80 \%$ of cases. On the other hand, abnormalities of repolarization mimicking an extended myocardial infarction remain more exceptional and constitute the originality of our observation.

\section{Conclusion:}

Diagnosis of myocarditis remains an embarrassing dilemma for clinicians especially in children as it can simulate myocardial infarction. 